\title{
ESTUDO DA ADSORÇÃO DA PROTEÍNA BSA EM HIDRÓXIDOS DUPLOS LAMELARES NANOESTRUTURADO
}

\author{
S. O. Santos ${ }^{1}$, P. A. S. Tavares ${ }^{1}$, K. A. B. Nogueira ${ }^{1}$, J. E. Aguiar ${ }^{1}$ e I. J. Silva Jr. ${ }^{1}$ \\ ${ }^{1}$ Universidade Federal do Ceará, Departamento de Engenharia Química \\ E-mail para contato: ivanildo@gpsa.ufc.br
}

\begin{abstract}
RESUMO - Este trabalho teve por objetivo sintetizar nanoestruturas de HDL e avaliar a adsorção da proteína BSA. O HDL foi sintetizado através do método de co-precipitação a pH variável. Logo depois o material foi analisado e caracterizado. Foram feitos estudos em batelada, utilizando tanques agitados para avaliar o efeito do $\mathrm{pH}$, da cinética e as isotermas de adsorção. De acordo com os resultados, a adsorção de BSA ocorreu preferencialmente no $\mathrm{pH}$ 4,8 em HDL $\mathrm{Mg} / \mathrm{Al}$ e em pH 6,7 em HDL Zn/Al. Os modelos de Langmuir e Langmuir Frendlich se ajustaram bem aos dados experimentais, obtendo parâmetros de equilíbrio. As quantidades máximas adsorvidas foram em torno de $180 \mathrm{mg} / \mathrm{mL}$ em HDL $\mathrm{Zn} / \mathrm{Al}$ e $139 \mathrm{mg} / \mathrm{mL}$ em HDL $\mathrm{Mg} / \mathrm{Al}$.
\end{abstract}

\section{INTRODUÇÃO}

Um campo de pesquisa que vem apresentando um aumento considerável nos últimos anos é o de desenvolvimento de materiais híbridos, devido principalmente ao fato de combinar o conhecimento já tradicional com novas abordagens e tecnologias modernas (Silva, et al., 2015). Os Hidróxidos duplos lamelares (HDL), por conter um grande potencial de aplicação em processos de separação (Zhang et al., 2013), catálise (Wang et al., 2012), ciências biomédicas (Cao et al., 2011), dentre outras aplicações, têm sido foco de muitos estudos nas últimas décadas, por conta da ampla versatilidade de modificação e capacidade de variação de cations, os HDLs podem gerar compostos com novas propriedades térmicas, texturais e estruturais, os tornando ainda mais promissores.

Em razão da grande aplicabilidade desse material, bem como a escassez de publicações sobre o uso do HDL para adsorção de proteínas, esse trabalho tem por objetivo sintetizar e caracterizar nanoestruturas de $\mathrm{HDL} \mathrm{Zn} / \mathrm{Al}$ e $\mathrm{Mg} / \mathrm{Al}$, além de realizar estudos do $\mathrm{pH}$, cinética e isoterma de adsorção com a proteína BSA.

\section{MATERIAIS E MÉTODOS}

Albumina do Soro Bovino (BSA) foi obtida da Sigma-Aldrich (EUA). Os demais reagentes utilizados foram todos de grau analítico e para a preparação de todas as soluções utilizou-se água destilada.

A preparação dos HDLs foi feita através do método de co-precipitação (Aguiar et al., 2013) a pH variável, seguido por tratamento hidrotérmico a $80^{\circ} \mathrm{C}$. 
Para a síntese de HDL Mg/Al, foram misturadas as soluções de magnésio [0,0996 mol de $\left.\left(\mathrm{Mg}\left(\mathrm{NO}_{3}\right)_{2} \cdot 6 \mathrm{H}_{2} \mathrm{O}\right)\right]$ e de alumínio [0,0332 mol de $\left(\mathrm{Al}\left(\mathrm{NO}_{3}\right)_{3} \cdot 9 \mathrm{H}_{2} \mathrm{O}\right]$ em $100 \mathrm{ml}$ de água destilada mantendo a razão molar dos cátions de 3:1. Em seguida, essa solução foi adicionada gota a gota a uma solução do ânion $\left[0,249 \mathrm{~mol}\right.$ de $\left.\left(\mathrm{NaHCO}_{3}\right)\right]$ a ser intercalado e mantida a $60{ }^{\circ} \mathrm{C}$ sob agitação vigorosa. Para a síntese de $\mathrm{HDL} \mathrm{Zn} / \mathrm{Al}$, foram misturadas as soluções de zinco [0,0614 mol de $\left.\mathrm{zncl}_{2}\right]$ e de alumínio [0,0307 mol de $\left.\left(\mathrm{Al}\left(\mathrm{NO}_{3}\right)_{3} \cdot 9 \mathrm{H}_{2 \mathrm{O}}\right)\right]$ em $100 \mathrm{ml} \mathrm{de}$ água estilada mantendo a razão molar dos cátions de 2:1. Em seguida, essa solução foi adicionada gota a gota a uma solução do ânion $\left[0,153 \mathrm{~mol}\right.$ de $\left.\left(\mathrm{NaHCO}_{3}\right)\right]$ a ser intercalado e mantida a $60^{\circ} \mathrm{C}$ sob agitação vigorosa.

Para se obter as nanoestruturas de Hidróxido Duplo Lamelar, foi utilizado o método convencional e modificado de co-precipitação segundo (Wang et al., 2013).

Pelo ponto de carga zero do $\left(\left[\mathrm{ZnAl}_{2}(\mathrm{OH})_{16}\right] \mathrm{CO}_{3} .4 \mathrm{H}_{2} \mathrm{O}\right)$ ser no $\mathrm{pH}$ 6.0, foi utilizado um pH mais elevado intencionalmente (de 12), a fim de tornar a superfície das nanoestruturas de HDL carregadas negativamente. $\mathrm{O}$ pH da solução de precipitação foi mantida constante a 12 pela adição de $\mathrm{NaOH}$ solução $(3,4 \mathrm{M})$ gota a gota mantendo as condições básicas para aumento da taxa de formação do HDL e o desenvolvimento de todas as direções das nanoestruturas esférica, devido à força de repulsão entre as partículas carregadas positivamente do HDL primário e os ânions $\mathrm{Al}(\mathrm{OH})^{4}-, \mathrm{CO}_{3}{ }^{2-}, \mathrm{OH}-$, fazendo com que o crescimento fosse inibido, o que conduziu à formação de partículas em escala nanométrica. Todos os outros parâmetros de síntese foram mantidos inalterados conforme o método de obtenção do precursor.

\subsection{Caracterização Estrutural}

Medições de adsorção-dessorção de $\mathrm{N}_{2}(99,999 \%)$ a $77 \mathrm{~K}$ foram realizadas utilizando um equipamento de volumetria (Autosorb-1MP, Quantachrome Instruments). As amostras foram previamente desgaseificadas a uma temperatura de $150{ }^{\circ} \mathrm{C}$ durante $12 \mathrm{~h}$ e $0,5 \mathrm{~Pa}$. A área de superfície específica $\left(\mathrm{S}_{\mathrm{BET}}\right)$ das amostras foi estimada a partir do método de Brunauer Emmet e Teller-BET (Gregg, 1982), usando os dados de adsorção na gama de pressões relativa de 0,05-0,12, 0,05-0,18 e 0,05-0,23 para as amostras de HDL $\mathrm{Zn} / \mathrm{Al}$ e $\mathrm{Mg} / \mathrm{Al}$, respectivamente. $\mathrm{O}$ volume total de poros $\left(\mathrm{V}_{\mathrm{TP}}\right)$ foi obtido pela regra de Gurvich a uma pressão relativa de 0.985 . O método $\alpha$-plot (Gregg, 1982) foi usado para calcular volumes de microporos $\left(\mathrm{V}_{\mu \mathrm{P}}\right)$. $\mathrm{O}$ volume de mesoporos $\left(\mathrm{V}_{\mathrm{MP}}\right)$ foi obtido a partir da diferença entre $\mathrm{V}_{\mathrm{TP}}$ e a soma de $\mathrm{V}_{\mu \mathrm{P}}$ e $\mathrm{V}_{\mathrm{MP}}$. O método VBS para determinação da distribuição do tamanho de poro foi proposto por (Villarroel-Rocha et al., 2014) para amostras com geometrias de poros cilíndricos e esféricos. Este método, baseado no algoritmo de $\mathrm{BJH}$, considera mecanismos adequados de condensação capilar e evaporação (para poros cilíndricos e esféricos) nos mesoporos, por meio da equação de Kelvin. O método VBS modifica essa equação com a adição de um termo de correção $\left(f_{c}\right)$ para se obter o raio do poro.

\subsection{Estudos de Adsorção}

2.4.1. Efeito do $\mathrm{pH}$ e cinética de adsorção: Os ensaios de adsorção foram realizados em sistema de batelada em um sistema de agitação orbital (TECNAL, modelo TE-165) a 16 rpm e temperatura de $22^{\circ} \mathrm{C}$, todos realizados em duplicata. Para verificar a influência do $\mathrm{pH}, 20$ 
mg de adsorvente foi posto em contato com $3 \mathrm{~mL}$ de solução de BSA $(1,0 \mathrm{mg} / \mathrm{mL})$ nos seguintes tampões: pH de 4,0 - 4,8 tampão acetato, 6,0 - 6,7 tampão MÊS, 7,8 - 10,0 tampão TRIS e 10,7 tampão bicarbonato de sódio, todos na concentração de $50 \mathrm{mM}$. O sistema ficou em agitação constante durante um período de 2 horas. Para os ensaios de cinética de adsorção, utilizou-se a concentração de $1,0 \mathrm{mg} / \mathrm{mL}$ de $\mathrm{BSA}$ em $\mathrm{pH}$ já fixo, e foi variado o tempo de contato de 1 a 360 minutos. Após este período, todas as amostras foram recolhidas e centrifugadas em microcentrífuga ( CT-15000R CIENTEC) durante 10 min com rotação de $10000 \mathrm{rpm}$, e as absorbâncias das amostras foram medidas a $280 \mathrm{~nm}$ (Espectrofotômetro de UV-Vis Biomate 3, Thermo Scientific, EUA).

A quantidade de proteína adsorvida $(\mathrm{mg} / \mathrm{g})$ foi calculada através de um balanço de massa, descrito pela equação a seguir:

$$
q=\frac{\mathrm{V}_{\mathrm{SOL}}\left(\mathrm{C}_{0}-\mathrm{C}_{\mathrm{EQ}}\right)}{\mathrm{m}_{\mathrm{ads}}}
$$

onde $\mathrm{C}_{\mathrm{EQ}}$ é a concentração de proteína na fase líquida $(\mathrm{mg} / \mathrm{mL})$ em equilíbrio, $\mathrm{C}_{\mathrm{O}}$ é a concentração inicial de proteína na fase líquida $(\mathrm{mg} / \mathrm{mL}), \mathrm{m}_{\mathrm{ads}}$ é a massa de HDL $(\mathrm{g})$ e $\mathrm{V}_{\mathrm{SOL}}$ é o volume da solução $(\mathrm{mL})$ para o contato com o HDL.

2.4.2. Isotermas de adsorção: A faixa de concentrações da proteína variou de 0,5 a 6,0 $\mathrm{mg} / \mathrm{mL}$. Foram utilizados os modelos de Langmuir e Langmuir-Freudlich, descritos pelas equações abaixo (2) e (3), respectivamente. Para modelagem dos dados experimentais, foi utilizado o software Origin, Microcal, EUA.

$$
\begin{aligned}
& \mathrm{q}=\frac{\mathrm{q}_{\mathrm{m}} \mathrm{C}_{\mathrm{eq}}}{\mathrm{K}_{\mathrm{D}}+\mathrm{C}_{\mathrm{eq}}} \\
& \mathrm{q}=\frac{\mathrm{q}_{\mathrm{m}}\left(\mathrm{K}_{\mathrm{LF}} \mathrm{C}_{\mathrm{eq}}\right)^{n}}{1+\left(\mathrm{K}_{\mathrm{LF}} \mathrm{C}_{\mathrm{eq}}\right)^{n}}
\end{aligned}
$$

Em que $q_{m}(m g / m L)$ é a capacidade de adsorção máxima, $\mathrm{K}(\mathrm{mL} / \mathrm{mg})$ é definida como a constante de Langmuir, $C_{e q}$ representa a concentração de proteína na solução em equilíbrio, $K_{L F}$ e $n$ são as constantes de adsorção de Langmuir-Freundlich, onde são indicadores de intensidade e da capacidade de adsorção de ligação, respectivamente.

\section{RESULTADOS E DISCUSSÕES}

\subsection{Caracterização Estrutural}

A Figura 1 ilustra as isotermas experimentais de adsorção-dessorção de $\mathrm{N}_{2}$ a $77 \mathrm{~K}$ para os materiais em estudo. Os dois tipos de materiais, $\mathrm{HDL} \mathrm{Mg} / \mathrm{Al}$ e $\mathrm{Zn} / \mathrm{Al}$ apresentam isotermas Tipo IV de acordo com a classificação da IUPAC (Rouquerol et al., 1999), que são típicos de materiais mesoporos. As isotermas dos materiais mostram um ponto de inflexão híbrido de histerese dos tipos H1 e H3 (Rouquerol et al., 1999). No entanto, a histerese da amostra do 


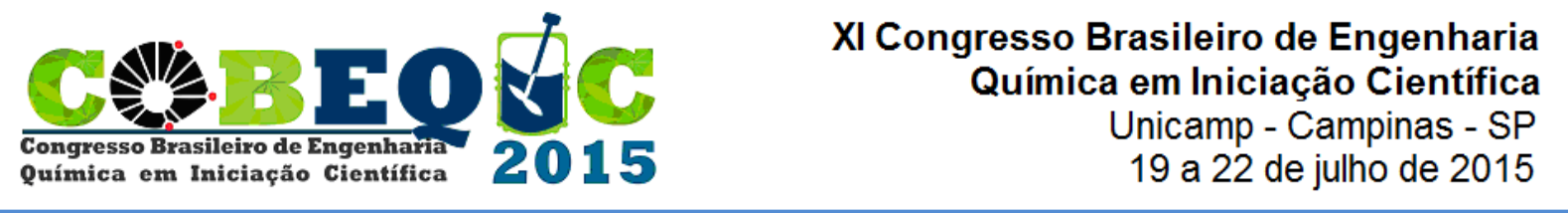

HDL $\mathrm{Zn} / \mathrm{Al}$ evidencia com maior clareza tipo histerese $\mathrm{H} 3$ característica de isotermas reversíveis a pressões de até de $0,45 \mathrm{P} / \mathrm{P} 0$.

Figura 1 - Isotermas experimentais de adsorção.

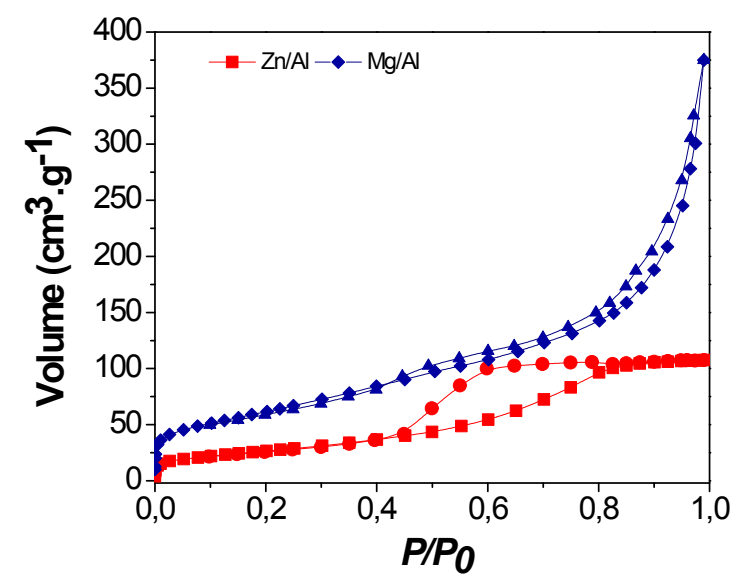

A Tabela 1 resume as características texturais dos materiais a base de $\mathrm{Mg}$ e $\mathrm{Zn}$, respectivamente.

Tabela 1 - Caraterizações texturais dos materiais.

\begin{tabular}{cccccc}
\hline Amostra & $\mathbf{S}_{\mathbf{B E T}} \mathbf{m}^{2} / \mathbf{g}$ & $\mathbf{V}_{\mu \mathrm{P}} / \mathrm{cm}^{3} / \mathrm{g}$ & $\mathbf{V}_{\mathrm{TP}} / \mathrm{cm}^{3} / \mathrm{g}$ & $\mathrm{V}_{\mathrm{MP}} / \mathrm{cm}^{3} / \mathrm{g}$ & $\mathrm{D}_{\mathrm{p}}(\mathrm{nm})$ \\
\hline$H D L \mathrm{Zn} / \mathrm{Al}$ & 100 & 0 & 0,17 & 0,17 & 6,8 \\
$H D L \mathrm{Mg} / \mathrm{Al}$ & 230 & 0 & 0,56 & 0,56 & 9,7 \\
\hline
\end{tabular}

As amostras mostraram uma predominância de mesoporosos obtidos pelo método VBS para todas as amostras em estudo com incidência de mesoporos cilíndricos e esféricos, característicos de materiais na base de sílica (Villarroel-Rocha et al., 2014).

\subsection{Estudos de Adsorção}

As curvas cinética e as isotermas de adsorção de BSA nos HDL $\mathrm{Mg} / \mathrm{Al}$ e $\mathrm{Zn} / \mathrm{Al}$ nanoestruturado estão representadas na Figura 2(a) e 2(b), respectivamente.

Os perfis cinéticos dos materiais (Figura 2(a)) mostram que ambos possuem um declínio rápido, porém, o $\mathrm{Mg} / \mathrm{Al}$ atingiu o tempo de equilíbrio mais rapidamente, por volta de $180 \mathrm{~min}$. Já para o $\mathrm{Zn} / \mathrm{Al}$, o tempo de equilíbrio foi atingido por volta dos $300 \mathrm{~min}$, e adsorvendo uma maior quantidade de BSA.

Observa-se que as quantidades adsorvidas aumentam à medida que se aumenta a concentração da proteína, atingindo o equilíbrio rapidamente, próximo à concentração de 2,0 $\mathrm{mg} / \mathrm{mL}$, em ambos os materiais. Percebe-se que a quantidade máxima adsorvida por $\mathrm{Zn} / \mathrm{Al}$ foi em torno de $180 \mathrm{mg} / \mathrm{mL}$ e em $\mathrm{Mg} / \mathrm{Al}$ foi cerca de $139 \mathrm{mg} / \mathrm{mL}$. Isso pode ser explicado pelo fato da síntese do HDL Mg/Al, onde a proporção dos cátions é de 3:1, diferente do $\mathrm{Zn} / \mathrm{Al}$ que é de 2:1. Por conter razão molar de 3:1, o HDL Mg/Al libera íons hidróxidos no meio, deixando a solução mais básica, e dificultando a adsorção. Portanto, as nanoestruturas de 
HDL $\mathrm{Zn} / \mathrm{Al}$ foram mais efetivas que as de Mg/Al. Os modelos de Langmuir e LangmuirFrendlich se ajustaram bem aos dados de isoterma, proporcionando os parâmetros mostrados na Tabela 2.

Figura 2. Cinética (a) e isotermas (b) de adsorção de BSA em HDL Zn/Al e Mg/Al. Modelos de Langmuir (-) e Langmuir-Freundlich (-) para ajuste de dados.

(a)

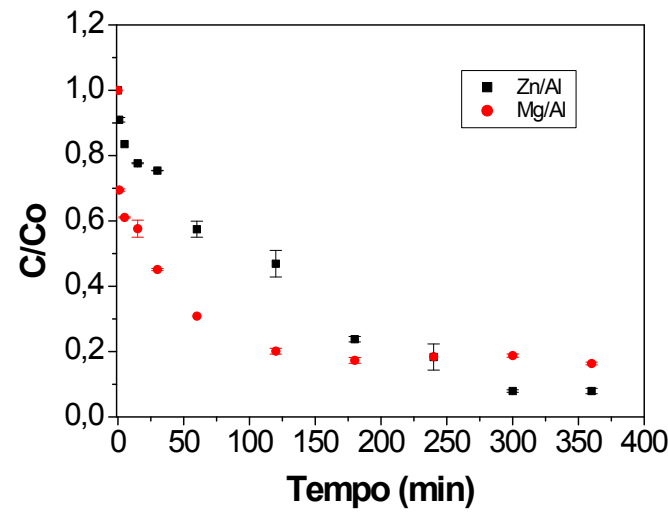

(b)

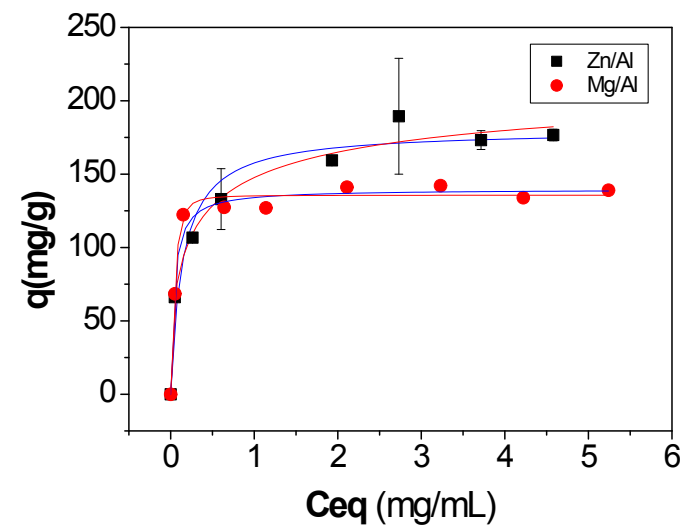

Tabela 2. Parâmetros de ajuste do modelo de Langmuir e Langmuir-Freundlich.

\begin{tabular}{ccccc}
\hline & \multicolumn{2}{c}{ HDL $(\mathbf{M g} / \mathbf{A l})$} & \multicolumn{2}{c}{ HDL $(\mathbf{Z n} / \mathbf{A l})$} \\
\cline { 2 - 5 } Parâmetros & L & L.F & L & L.F \\
\hline$q_{\max }(\mathrm{mg} / \mathrm{g})$ & 139.63 & 135.65 & 180.08 & 227.32 \\
$K(\mathrm{~mol} / \mathrm{L})$ & 23.92 & 0.0036 & 7.00 & 0.54 \\
$\chi^{2}$ & 51.23 & 35.22 & 178.65 & 80.20 \\
$\mathrm{R}^{2}$ & 0.98 & 0.99 & 0.96 & 0.99 \\
$b$ & - & 1.87 & - & 0.51 \\
\hline
\end{tabular}

Deve-se considerar ainda, que os mecanismos de adsorção de superfície devem-se às interações eletrostáticas entre elétrons deslocados em planos superficiais do HDL e elétrons livres das moléculas das proteínas. No caso das proteínas, deve-se levar em consideração o ponto isoelétrico $(\mathrm{pI})$, onde há um balanceamento igual das cargas da proteína, promovendo uma maior adsorção. Para tanto, a investigação da adsorção das nanoestruturas de HDL em diferentes condições de $\mathrm{pH}$ foi feita em diferentes soluções tampão, variando os valores de $\mathrm{pH}$. Os resultados obtidos variaram de um material para o outro. Para nanoestruturas de HDL $\mathrm{Mg} / \mathrm{Al}$, o melhor valor de $\mathrm{pH}$ obtido foi 4,8 , justo no $\mathrm{pI}$ da proteína. Já para nanoestruturas de HDL Zn/Al, o melhor valor de $\mathrm{pH}$ obtido foi 6,7, acima do $\mathrm{pI}$ da BSA. A força eletrostática aumenta a quantidade de proteína na superfície de HDL, provavelmente devido a uma interação mais forte entre a carga negativa da BSA e a carga positiva de HDL. Portanto, Zn é mais eletropositivo que o $\mathrm{Mg}$ e, dessa forma, mais reativo. 


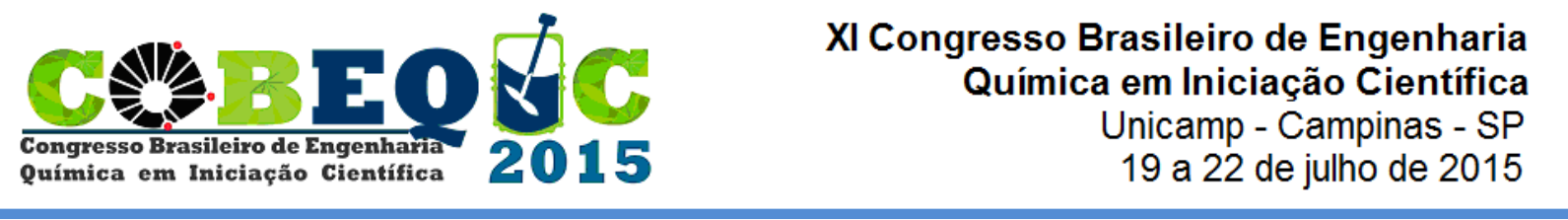

\section{CONCLUSÃO}

Através dos resultados obtidos, percebeu-se que as quantidades adsorvidas da proteína do soro bovino BSA se mostrou mais elevada em nanoestruturas de HDL Zn/Al. Os modelos de Langmuir e Langmuir-Frendlich se ajustaram bem aos dados proporcionando quantidade máxima adsorvida em torno de $180 \mathrm{mg} / \mathrm{mL}$ para nanoestruturas de $\mathrm{HDL} \mathrm{Zn} / \mathrm{Al}$ e $139 \mathrm{mg} / \mathrm{mL}$ para $\mathrm{Mg} / \mathrm{Al}$. Isso pode ser explicado através da síntese dos dois materiais, que ocorre em razões molares diferentes. Portanto, as nanoestruturas de HDL Zn/Al se mostraram mais efetivas na adsorção de BSA. Mais estudos devem ser feitos, a fim de comprovar a eficácia do material.

\section{REFERENCIAS}

AGUIAR, J. E.; BEZERRA, B. T. C.; BRAGA, B. M.; LIMA, P. D. S.; NOGUEIRA, R. E. F. Q.; LUCENA, S. M. P.; SILVA JR., I. J Adsorption of Anionic and Cationic Dyes from Aqueous Solution on Non-Calcined Mg-Al Layered Double Hydroxide: Experimental and Theoretical Study, Separ Sci. Technol. v. 48, p. 2307-2316, 2013.

$\mathrm{CAO}, \mathrm{F}$. et al. Zn-Al-NO3-layered double hydroxides with intercalated diclofenac for ocular delivery. International Journal of Pharmaceutics, v. 404, n. 1-2, p. 250-256, 2011.

GREGG, S. J. A. S., K.S.W. Adsorption, surface area and porosity. second ed. New York: Academic Press, 1982. 303.

ROUQUEROL, J.; ROUQUEROL, F.; SING, K. S. W. Adsorption by powders \& porous solids. San Diego: 1999. 467.

S.R.P. SILVA, M.J. BELIATIS, K.D.G.I. JAYAWARDENA, C.A. MILLS, R. RHODES, L.J. ROZANSKI. Hybrid and nanocomposite materials for flexible organic electronics applications. Handbook of Flexible Organic Electronics, 2015, Pages 57-84

VILLARROEL-ROCHA, J.; BARRERA, D.; SAPAG, K. Introducing a self-consistent test and the corresponding modification in the Barrett, Joyner and Halenda method for pore-size determination. Microporous and Mesoporous Materials, v. 200, n. 0, p. 68$78,2014$.

WANG, Q.; OHARE, D. Recent advances in the synthesis and application of layered double hydroxide (LDH) nanosheets. Chemical Reviews, v. 112, n. 7, p. 4124-4155, 2012.

WANG, K.-Y. et al. Layered indium chalcogenidoantimonates [Me2NH2]2In2Sb2S7-xSex (x $=0,2.20,4.20,7)$ with tunable band gaps and photocatalytic properties. CrystEngComm, v. 14, n. 1, p. 90-94, 2012.

ZHANG, T. et al. Biomimetic fabrication of hierarchically structured LDHs/ZnO composites for the separation of bovine serum albumin. Chemical Engineering Journal, v. 219, n. 0 , p. 278-285, 2013. 